\title{
Orbital Clustering in the Distant Solar System
}

\author{
Michael E. Brown (DD and Konstantin Batygin \\ Division of Geological and Planetary Sciences, California Institute of Technology, Pasadena, CA 91125, USA; mbrown@caltech.edu, kbatygin@gps.caltech.edu \\ Received 2018 July 27; revised 2018 October 17; accepted 2018 November 11; published 2019 January 22
}

\begin{abstract}
The most distant Kuiper Belt objects (KBOs) appear to be clustered in longitude of perihelion and in orbital pole position. To date, the only two suggestions for the cause of these apparent clusterings have been either the effects of observational bias or the existence of a distant giant planet in an eccentric inclined orbit known as Planet Nine. To determine if observational bias can be the cause of these apparent clusterings, we develop a rigorous method of quantifying the observational biases in the observations of longitude of perihelion and orbital pole position. From this now more complete understanding of the biases, we calculate that the probability that these distant KBOs would be clustered as strongly as observed in both longitude of perihelion and in orbital pole position is only $0.2 \%$. While explanations other than Planet Nine may someday be found, the statistical significance of this clustering is now difficult to discount.
\end{abstract}

Key words: Kuiper belt: general

\section{Introduction}

The apparent physical alignment of the perihelion position and orbital poles of many of the most distant Kuiper Belt objects (KBOs) has been taken to be evidence of a massive eccentric inclined planet well beyond Neptune (Batygin \& Brown 2016). To date no successful alternative dynamical explanation for such clustering has been suggested. Instead, discussion has focused on the possibility that the apparent physical alignments might be due to either random chance or highly affected by observational bias or both. Observational bias for distant objects is of critical concern. Objects on these extremely eccentric orbits are often found close to perihelion, so the sky distribution of observational surveys has a strong effect on the distribution of observed values of longitude of perihelion. Observations near the ecliptic preferentially find objects with longitude of ascending node close to the longitude of the observation, providing a strong potential bias to the pole position, too.

In Batygin \& Brown (2016), we made a simple estimate of the possibility of observational bias by suggesting that the biases in the discoveries of objects with semimajor axes beyond 230 au should not differ significantly from those of discoveries from 50 to $230 \mathrm{au}$. We found that under this assumption, the probability that the clustering of the then six known objects beyond $230 \mathrm{au}$ was highly significant. Given this simplistic initial assumption about observational biases and the importance of understanding this potential clustering, Brown (2017) developed a more rigorous method of estimating the longitude of perihelion bias by using the collection of all reported $\mathrm{KBO}$ discoveries to back out the probabilities that distant eccentric objects could have been detected in the collection of all surveys to date. No reliable method was available for evaluating the biases in pole position, however, rendering this work incomplete.

Conversely, calculation of the full set of observational biases for the well calibrated OSSOS survey found that their discovery of four objects with semimajor axis beyond $230 \mathrm{au}$ was consistent with a uniform distribution of orbital angles (Shankman et al. 2017), in apparent conflict with the clustering observed elsewhere. Unfortunately, although OSSOS collected by far the largest number of well characterized discoveries of KBOs, the survey was limited to two regions of ecliptic longitude, and these two longitudes are nearly orthogonal to the apparent longitude of perihelion clustering. As a result, the strength of the constraint on clustering from OSSOS is not yet clear, and will be analyzed below.

The lack of an explicit calculation of the bias in pole position in Brown (2017) remains a key impediment to a full treatment of the effect of observational biases on the observed clustering of distant objects. Here we extend the technique developed in that paper (which is in itself an extension of the technique developed in Trujillo \& Brown 2001) to develop a method to fully include both longitude of perihelion and orbital pole position in our bias calculations. We use these full bias calculations to determine the probability that a randomly distributed set of distant objects would be simultaneously clustered in longitude of perihelion and in pole position as strongly as the observations suggest.

\section{Longitude of Perihelion Bias}

Before developing our general method, we first revisit and update the longitude of perihelion bias. As of 2018 July 1, there are 14 known objects with perihelion beyond Neptune and semimajor axis beyond $230 \mathrm{au}$. We calculate the longitude of perihelion bias for each object independently using the method of Batygin \& Brown (2016), in which we use the entire MPC database of reported KBO discoveries (again, as of 2018 July 1) to determine probabilities of discovering distant KBOs as a function of longitude of perihelion. Previously, we calculated the significance of the clustering by determining the Rayleigh $Z$ statistic, taking each longitude of perihelion, projecting a unit vector in that direction, taking the two-dimensional average of all of the vectors, and assessing the significance of the length of the mean vector. Here we slightly modify this procedure.

Instead of projecting a unit vector into the geometrical direction of the longitude, we instead employ canonically conjugated variables to more properly represent the orbital parameters. Starting with the reduced Poincaré action-angle 


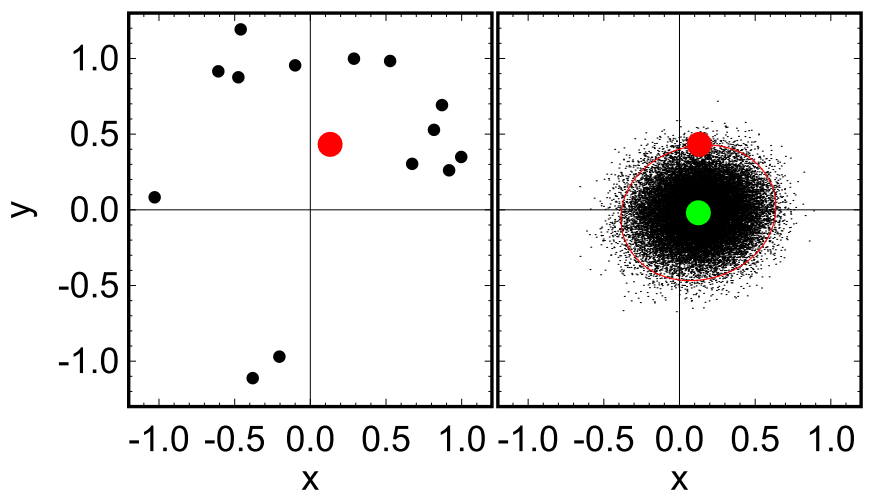

Figure 1. (a) The canonical $(x, y)$ coordinates for the 14 known KBOs with semimajor axis greater than $230 \mathrm{au}$. These coordinates point in the direction of the longitude of perihelion of each object but are modulated by a function of eccentricity. The average position of $(0.13,0.43)$ is marked in red. The distance away from the origin of this average is a measure of the strength of the clustering. (b) Average $(x, y)$ positions of each of 100,000 iterations in which we randomly select longitudes of perihelion from the bias distribution functions for each of the 14 objects (under the assumption that the longitudes are distributed uniformly) and then compute the average $(x, y)$ position of the 14 random objects of each iteration. The average $(x, y)$ position of the 14 real distant objects (red point) is more strongly clustered than $96 \%$ of the random iterations where the longitudes are assumed to be uniform (enclosed within the red ellipse). Overall, a population of objects that was uniform in longitude of perihelion would be biased on average to have a longitude of perihelion clustering toward $-7^{\circ}$, shown as a green point.

coordinates (e.g., Morbidelli 2002)

$$
\begin{gathered}
\Gamma=1-\sqrt{1-e^{2}} \quad \gamma=-\varpi \\
Z=\sqrt{1-e^{2}}(1-\cos (i)) z=-\Omega .
\end{gathered}
$$

(here we have scaled $\Gamma$ and $Z$ by $\Lambda=\sqrt{G M_{\odot} a}$, as this factor does not affect our analysis), we define our clustering in terms of canonical Cartesian analogs of Poincaré variables, defined as:

$$
\begin{aligned}
& x=\sqrt{2 \Gamma} \cos (\varpi) y=\sqrt{2 \Gamma} \sin (\varpi) \\
& p=\sqrt{2 Z} \cos (\Omega) q=\sqrt{2 Z} \sin (\Omega) .
\end{aligned}
$$

In practice, the $(x, y)$ coordinate of an object is simply a vector in the direction of the longitude of perihelion, properly scaled by a function of eccentricity, and the $(p, q)$ coordinate is a projection of the orbital pole position of the object (for small values of $i$ ) also properly scaled by a function of eccentricity. We will thus use the clustering of these vectors as the appropriate measures for the clustering of the longitude of perihelion and the pole position. As all of the eccentricities of the distant objects are between 0.69 and 0.98 , using the $(x, y, p$, $q$ ) coordinate system rather than the simple angle of the longitude of perihelion and the projected pole position makes little difference to the final result, but an advantage of adopting these coordinates is that by virtue of the Poisson bracket criterion, $(x, y, p, q)$ provides an orthogonal basis set for representing orbital parameters. Below, we will combine the longitude of perihelion and pole position clustering into a single value in $(x, y, p, q)$ space, necessitating this orthogonal basis.

With the appropriate variables defined, we return to computing the bias in the longitude of perihelion. Figure 1(a) shows the position of each of the 14 known objects in $(x, y)$ space. The average of these position is $(0.13,0.43)$, corresponding to an average clustering in longitude of perihelion in the

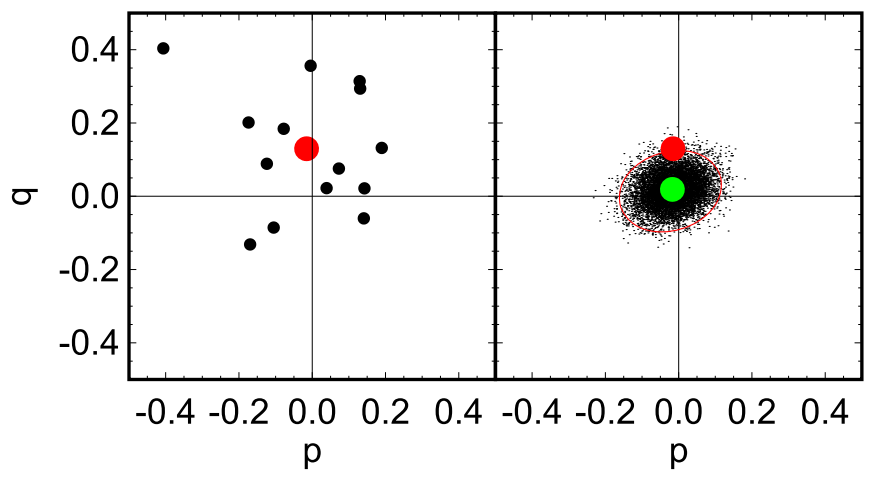

Figure 2. (a) The canonical $(p, q)$ coordinates for the 14 known KBOs with semimajor axis greater than 230 au. These coordinates point in the direction of the longitude of ascending node with a magnitude of approximately $\sin i$, but modulated by a function of eccentricity. The average position of $(-0.02,0.13)$ is marked in red. (b) Average $(p, q)$ positions of 100,000 iterations in which we randomly select pole positions for the 14 distant KBOs using the bias distribution functions and assuming a uniform distribution of node and a scattered disk-like distribution in inclination and calculate the average $(p, q)$ position for each iteration. The average $(p, q)$ position of the 14 real distant objects (red point) is more strongly clustered than $96.5 \%$ of the random iterations (enclosed in the red ellipse). Overall, the observations are biased to have a pole position bias along a line of nodes from $17^{\circ}$ to $197^{\circ}$ as can be seen from the elongation of the cloud of points along that line.

direction of $\varpi=73^{\circ}$. The distance from the origin of this average position shows the strength of the clustering, in analogy to the Rayleigh $\mathrm{Z}$ test, though because these are not unit vectors, we cannot use the $\mathrm{Z}$ test and must develop a modified measure of significance. In order to calculate the significance of this observed clustering, then, we perform 100,000 iterations in which we select a random longitude of perihelion for each of the 14 objects, sampling from the calculated bias distribution for each object. For each iteration, we then calculate the average $(x, y)$ coordinate of the 14 randomly selected objects (Figure 1(b)). This distribution of average positions from the 100,000 iterations shows the probability that observations of a population that was uniformly distributed in longitude of perihelion would have a given average clustering.

From the distribution of these random iterations, it is apparent that there is an average bias of $\varpi$ toward $\sim-7^{\circ}$, nearly perpendicular to the actual clustering detected. Because the bias is not symmetric with $\varpi$, we calculate the probability that the clustering of the real objects would be as strong as seen and in the direction observed by finding the minimum-area two-dimensional ellipse, which encloses the maximum number of random iteration averages and also the real data point. The red ellipse in Figure 1(b) encompasses $96 \%$ of the random iterations. We find that only $4 \%$ of random iterations are clustered as significantly in longitude of perihelion as the real data.

\section{Orbital Pole Bias}

The distant KBOs also appear to be clustered in orbital pole position. In Figure 2(a) we show the positions of the 14 known distant objects in $(p, q)$ space, which essentially shows the orbital pole positions of the objects. The average position of $(-0.02,0.13)$ is marked.

As with the longitude of perihelion debiasing, a full debiasing of the observations to account for pole position would require a detailed knowledge of all surveys that have 
detected KBOs. With rare exception, such information is not available. Instead, we simply have lists of reported objects that include the derived orbital parameters, brightness, and detection location of the object. We extend the technique developed in Brown (2017) to use this information to compute an objectby-object debiasing of the pole positions and inclinations of the distant objects.

We use the fact that a survey that discovered a $\mathrm{KBO}$ with a particular brightness at a particular location would have been equally sensitive to any $\mathrm{KBO}$ at least as bright as the detected object if it had been at that position at that time (we discuss important caveats to this assumption below). Our procedure is thus as follows. For each individual distant $\mathrm{KBO}$, we go through every known discovered $\mathrm{KBO}$ and ask which siblings of the distant $\mathrm{KBO}$ could have been discovered at the position of the discovered KBO. We define the siblings to be all potential KBOs with the same absolute magnitude, $a, e$, and $\varpi$ as the distant object, but that have the same sky position as the KBO discovery. We allow the siblings to be uniformly distributed in the direction of orbital motion around the discovery position. The values of $\Omega, i$, and mean anomaly, $M$ for the sibling are uniquely defined by the sky position of the discovered KBO and its direction of orbital motion. For the derived value of $M$, we calculate the heliocentric distance and thus the relative magnitude that the sibling $\mathrm{KBO}$ would have at that location. If the sibling $\mathrm{KBO}$ is bright enough that it would have been discovered at that location (that is, it is as bright or brighter than the true discovered KBO), we add one to the cell position of an equal area grid on the sky corresponding to the pole of the sibling KBO (implemented using the IDL HEALpix library ${ }^{1}$ ). Repeating this process for all detected KBOs builds a map that shows the relative probability of detection of the distant $\mathrm{KBO}$ under the assumption that pole positions are uniform across the sky. We then repeat the entire process for each individual distant $\mathrm{KBO}$ to build individual probability distributions.

This debiasing method relies on the assumption that KBOs discovered are a fair representation of the surveys that have been carried out and that the surveys could have detected any sibling $\mathrm{KBO}$ brighter than the real detections. Important caveats and corrections to these assumptions, including corrections for $\mathrm{KBO}$ distance, longitudinal clustering of resonant KBOs, and the latitudinal surface density of KBOs are discussed in Brown (2017). We test the sensitivity of the results to each of these assumptions and find that the results do not depend strongly on any of the precise assumptions. We add one modification to that analysis. The large survey of Sheppard et al. (2016) is dedicated to finding distant objects. As such, only objects with initial discovery distances greater than $50 \mathrm{au}$ are tracked. The actual survey, then, covers a much larger area than would be inferred from the reported discoveries. We thus weight each object discovered by Sheppard et al. (2016) by the ratio of the expected number of objects that they would have discovered had they tracked the closer objects to the number of distant objects they actually detected. In detail, we determine this ratio by noting that the number density of scattered disk objects (which comprise a majority of objects with discovery distance $r>50 \mathrm{au}$ ) as a function of latitude is approximately proportional to the number density of hot classical KBOs as a function of latitude (Brown 2001); so, the appropriate

\footnotetext{
https://healpix.jpl.nasa.gov/html/idl.htm
}
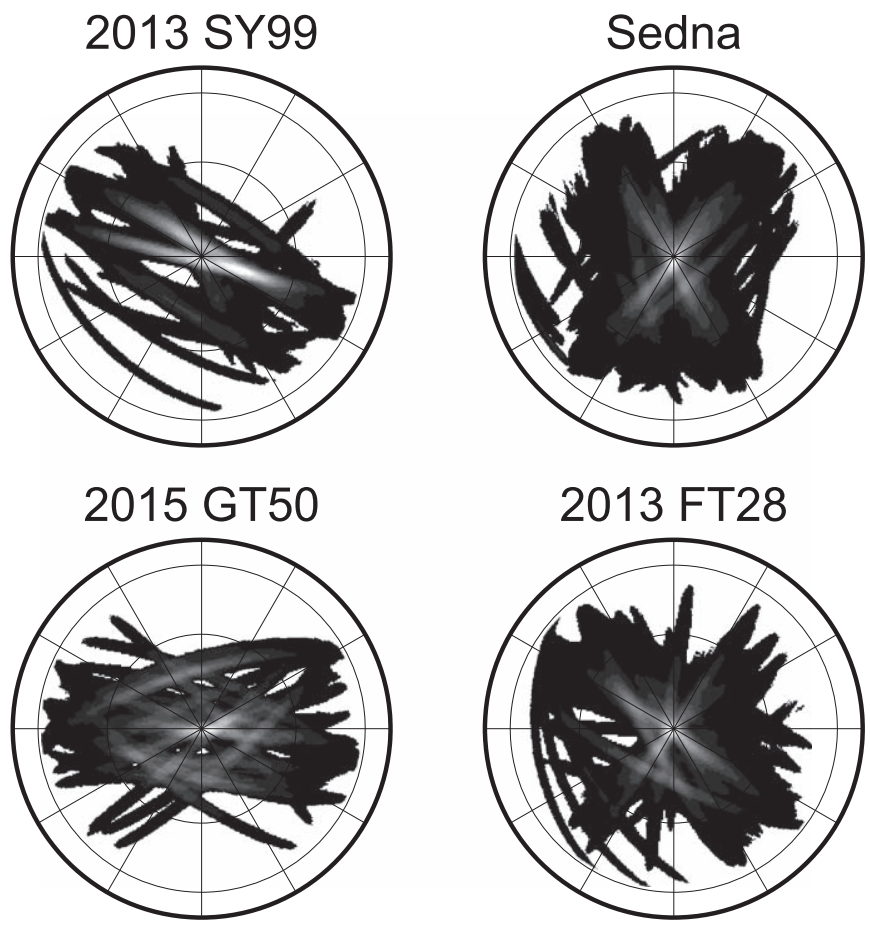

Figure 3. The probability of detection of a sibling KBO to a distant KBO, as a function of the sibling orbital pole position, shown as a polar projection. Radial grid lines are shown every $30^{\circ}$ in ascending node, with $\Omega=0$ at the right, and grid circles are shown every $30^{\circ}$ of inclination, with zero inclination at the center.

weighting is the total number of known objects (with $a>40$ and $r>30 \mathrm{au}$, as discussed in Brown (2017)) discovered at absolute heliocentric latitudes greater than $5^{\circ}$ (to exclude the cold classical objects) divided by the total number of these objects discovered at these latitudes but with $r>50 \mathrm{au}$, in both cases excluding objects discovered by the Dark Energy Camera (DEC), which was used for the survey (note that all DEC discoveries are excluded because it is not possible to always determine which specific survey made a specific discovery). We find 362 total objects with $r>30$ au and 35 with $r>50 \mathrm{au}$, for a ratio of $10 \pm 2$. The results are again insensitive to the precise value used.

The probability distribution functions for the pole positions of four representative distant KBOs are shown in Figure 3. The pole positions of discoveries of distant KBOs are heavily biased. Deep limited area surveys like OSSOS and the Dark Energy Survey contribute specific high-probability bands in the maps that are easily identified. Surveys that cover the sky more uniformly have their probabilities more distributed and, while they contribute heavily to the overall probability distribution, they do not stand out as clearly.

While the biases are severe, no clear bias toward a pole offset in the observed direction is obvious. We assess the likelihood that observational biases lead to the clustering in pole position by performing 100,000 iterations of a test where we choose a random object from the inclination distributionweighted probability distribution of each of the 14 objects and then calculate the average $(p, q)$ coordinates for each iteration.

As with the longitude of perihelion clustering, the observed clustering in $(p, q)$ space of the 14 distant object appears stronger than most of the iterations in which we assume a uniform distribution of longitudes of ascending node. To determine the significance of this clustering, we again fit a 
minimum-area two-dimensional ellipse to maximize the number of included randomly chosen $(p, q)$ points and to also include the real observed clustering. The red ellipse in Figure 2(b) encloses $96.5 \%$ of the random points. Only $3.5 \%$ of the random iterations are as strongly clustered in pole position as the real observations. As can be seen (Figure 2(b)), a bias exists toward pole positions with longitudes of ascending nodes along a line from $\sim 17^{\circ}$ to $\sim 197^{\circ}$, the approximate longitudes of the OSSOS survey regions.

\section{Combining Longitude of Perihelion and Pole Position Bias}

The probability that both longitude of perihelion and pole position would be clustered is significantly lower than that of either one independently. We use our previously developed method to extend our bias analysis to explicitly calculate the probability of finding an object with both a given longitude of perihelion and pole position. To perform this extension, we simply take our pole position bias method, which required that the object have a longitude of perihelion equal to that observed, and recalculate the pole position bias as a function of a (assumed to be uniform) longitude of perihelion, which we discretize into one degree bins. For each of the 14 observed objects, with observed values of $a, e$, and $H$, we now explicitly have the probability distribution function that such an object would have been discovered with a given longitude of perihelion and pole position under the assumption that the underlying distribution is uniform in longitude of perihelion and in pole position.

While a uniform longitude of perihelion is the correct assumption against which to test, we clearly do not want to assume a uniform distribution of poles. Instead, we assume a uniform distribution in longitude of ascending node and seek a realistic distribution in inclination. We examine the inclinations of distant KBOs using the method developed by Brown (2001). Perhaps the most appropriate inclination distribution to assume for our objects would be the inclination distribution of the entire scattered disk. We find that the 149 multi-opposition objects with $a>50$ au and $q>30$ au are fit by a standard function of $f(i) \propto \sin (i) \exp \left(-i^{2} / 2 \sigma^{2}\right)$ with $\sigma=14^{\circ} .9 \pm 0.6$. It is possible that the more distant objects have a different inclination distribution, however. If we fit only the 27 multiopposition objects with $a>150$ au and $q>30$ au, we find a best fit of $\sigma=15^{\circ} \pm 2^{\circ}$, consistent with the larger sample. Finally, we examine just the 14 objects with $a>230$ au and $q>30$ au. Here, we find a slightly lower inclination distribution of $\sigma=11_{-2}^{+3}$ degrees. In the significance analysis below, a narrower inclination distribution will make a given pole position clustering appear more significant, as a narrow inclination distribution is less likely to have an average far from the ecliptic pole. Thus, to be conservative, we use $\sigma=16^{\circ}$-larger than any of the best-fit values- as our assumed inclination distribution.

With the full probability distribution calculated, we now perform 1.6 million iterations where we randomly choose a longitude of perihelion and a pole position from the probability distribution function for each of the 14 objects and calculate the average of those 14 objects in $(x, y, p, q)$ space. We perform a four-dimensional analog to the ellipse fitting of Figures $1 \& 2$ and find that only $99.8 \%$ of iterations have an average as extreme at the real measured values of the distant objects. This

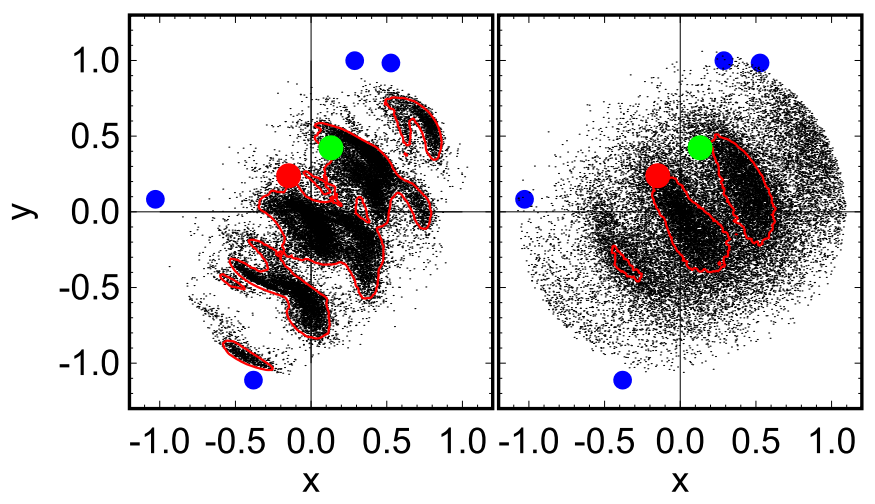

Figure 4. The canonical $(x, y)$ coordinates for the four distant OSSOS discoveries (blue dots). The mean of the OSSOS discoveries is shown as a red dot, while the mean of the full set of 14 objects is shown as the green dot. (a) $1,00,000$ iterations of randomly sampling our derived OSSOS biases and calculating a mean $(x, y)$ value. The mean values are patchy because there are only four objects and the biases are strong in two opposite directions. The red contours enclose $85 \%$ of the points and intersect the OSSOS mean. Only $15 \%$ of the random iterations are more clustered than the real OSSOS objects, an approximately $1 \sigma$ effect. (b) 1,00,000 iterations of randomly sampling using the published average OSSOS biases, rather than constructing and object-byobject bias. The large spread in longitude of perihelion positions (compared to Figure 1(b), for example) shows the relative insensitivity of OSSOS to longitude of perihelion clustering compared to using the full data set. A clustering as strong as that detected in the full data set (green dot) could only be detected at the $65 \%$ confidence level using the OSSOS survey. Because of the limited survey region and small number of detected distant objects, OSSOS observations are equally consistent with being drawn from a uniform distribution of longitudes of perihelion or with being clustered in longitude of perihelion as strongly as seen in the ensemble data.

number corresponds to a probability of finding the combined longitude of perihelion and pole cluster of only $0.2 \%$.

\section{Comparison to OSSOS}

The OSSOS observations of four distant KBOs have been suggested to be consistent with a uniform distribution of longitudes of perihelion (Shankman et al. 2017). We examine whether the severe observational selection effects of OSSOS coupled with the small number of distant objects discovered might prevent them from detecting the longitude of perihelion clustering seen here.

Figure 4 shows the $(x, y)$ positions of the four OSSOS objects (blue points). These objects have an average $(x, y)$ of $(-0.14,0.24)$, corresponding to an average longitude of perihelion of $140^{\circ}$ (red point), similar to the average $(x, y)$ value from the full data set of 14 objects (green point). To understand the biases for these objects from the OSSOS survey alone, we use the method developed above. We create longitude of perihelion bias distributions for the four distant OSSOS discovered objects using only the KBOs discovered in the OSSOS survey as our observational set. Because of the limited range of ecliptic longitudes of OSSOS, the biases for the individual objects are significantly more severe than found when using the complete catalog set. We then perform 100,000 random iterations as above, where we randomly select longitudes of perihelion for the four OSSOS objects from the bias distribution developed from the full set of OSSOS objects. Figure 4(a) shows the average $(x, y)$ position of the four objects from each of the 100,000 iterations. Where similar results for the full data set showed a smooth distributions of points (Figure 1(b)), the distribution here is quite clumpy. This clumpiness is driven by the strong biases in the potential 
discovery longitudes of perihelion in the OSSOS survey and the small number of objects being averaged. In spite of this clumpiness, it can be seen that a strong bias exists for the average longitude of perihelion to be somewhere along an axis from $\sim 45^{\circ}$ to $\sim 225^{\circ}$. Interestingly, the mean $(x, y)$ position of the four OSSOS discoveries is displaced in the orthogonal direction to the strong biases. A two-dimensional ellipse is a poor representation of the spread of the data in this case, so we instead draw contours enclosing the regions of highest density. We find that only $15 \%$ of the random iterations are more strongly clustered than the four real objects, an approximately $1 \sigma$ effect. These four objects cluster approximately in the same direction as the clustering seen in the full data set.

Shankman et al. (2017) calculate biases differently. Rather than evaluating the biases on an object-by-object basis, considering the specific orbital elements of each object, they instead assume a specific distribution for the orbital elements of distant objects and generate biases for this assumed distribution. We evaluate how this alternate method affects the longitude of perihelion bias. We use the reported OSSOS derived average bias function of Shankman et al. (2017) and repeat our analysis with a separate set of average $(x, y)$ positions found in 100,000 iterations (Figure 4(b)). This average set of $(x, y)$ positions is smoother, as expected from using a single average bias, though with approximately the same overall distribution as above. Here, we find that the OSSOS observed clustering is no longer significant, as the random spread in longitude of perihelion has increased to be larger than the measured clustering. More importantly, we find that even if a clustering as strong as that observed in the full data set were present (green point in Figure 4), it could only be detected at the $65 \%$ confidence level. That is, the uncertainties in the measurement of clustering from the OSSOS data are so large that OSSOS would not be capable of confidently detecting the clustering seen in the larger data set even if it were real and present in the OSSOS data. Because of the limited survey region and small number of detected objects, OSSOS observations are equally consistent with being drawn from a uniform distribution of longitudes of perihelion and with being clustered in longitude of perihelion as strongly as seen in the ensemble data. No conclusions on clustering of longitude of perihelion observed in the complete data set can be drawn from the OSSOS data.

\section{Conclusions}

Fully taking into account the biases in longitude of perihelion, inclination, and longitude of ascending node, the probability that the 14 known KBOs with semimajor axes beyond $230 \mathrm{au}$ would be clustered as strongly as currently observed due only to a combination of observational bias and random chance is only $0.2 \%$. While it remains true that the OSSOS survey is consistent with a uniform distribution of these parameters, we have shown that the severe longitudinal bias of the OSSOS survey renders it insensitive to the clustering observed in the more evenly distributed surveys. While the existence of Planet Nine remains the only current hypothesis for the explanation of this clustering (Batygin \& Brown 2016), we have shown that the joint clustering in longitude of perihelion and pole position of the distant KBOs is nearly indisputable, regardless of the existence of Planet Nine. If Planet Nine is not responsible for this clustering, new dynamical processes need to be found in the outer solar system.

We would like to thank the referee for an insightful question that lead to a significant improvement in this analysis. Discussions with David Gerdes, Matt Holman, Chad Trujillo, and Elizabeth Bailey helped to shape these arguments.

\section{ORCID iDs}

Michael E. Brown (ib https://orcid.org/0000-0002-8255-0545

\section{References}

Batygin, K., \& Brown, M. E. 2016, AJ, 151, 22

Brown, M. E. 2001, AJ, 121, 2804

Brown, M. E. 2017, AJ, 154, 65

Morbidelli, A. 2002, Modern Celestial Mechanics, Dynamics in the Solar System (Boca Raton, FL: CRC Press)

Shankman, C., Kavelaars, J. J., Bannister, M. T., et al. 2017, AJ, 154, 50

Sheppard, S. S., Trujillo, C., \& Tholen, D. J. 2016, ApJL, 825, L13

Trujillo, C. A., \& Brown, M. E. 2001, ApJL, 554, L95 